\title{
Allocating Aircraft Repairable Spare Parts Inventory for Single Training Base of a Flight University
}

\author{
Huimin Tang ${ }^{1 a^{*}}$ \\ ${ }^{1}$ Civil Aviation Flight University of China, Guanghan, Sichuan, China \\ a342674675@qq.com
}

Keywords: Logistics; Spare Parts; Repairable Parts; Multi-item

\begin{abstract}
Aircrafts' repairable spare parts inventory control has long been received great importance over the decades. In order to allocate the critical spare parts inventory subjected to finite investment constraints, a single location multi-item model is deployed to decide the base stock of each different training base in a flight university. Then a marginal analysis is used to generate the results, showing that the method is suitable and reasonable in this real life system.
\end{abstract}

\section{Introduction}

Modern equipments are designed increasingly complicated with more components. It placed bigger challenges to maintenance. Especially in aviation industry, the failures of key components will result in aircraft disruption if not having enough spare parts in stock. Due to long lead time in repair and transport, commercial airlines suffer from flight delay or cancellation, thus reduction of revenue and loss of reputation. While in aviation training industry, managing spare parts inventory also accounts for great importance. For example, at a flight university in China, downtimes of aircrafts will suspend normal flight training, which in turn delay many pilot students' graduation.

Over the last several decades, researchers and scholars have developed a great number of inventory models to deal with repairable parts problems. In 1966, Feeney and Sherbrooke [1] first formulated basic model to decide the level of single-item repairable parts inventory. Then Sherbrooke[2, 3] proposed a METRIC model for US air force to determine the backorders in a two-echelon inventory system. It's the first approach to maximize the availability of equipments in a system-oriented way, and a variety of such models came out afterwards. Muckstadt [4] proposed MOD-METRIC model to control the inventory of service parts in a multi-echelon and multi-order environment. Then VARI-METRIC model was put forward by Slay[5]. These models are modified to better fit to real life multi-echelon systems. But some models can only be derived by approximated solutions because of increasing complexity of its functions.

\section{Problem Description}

The flight university established 60 years ago and is well-known as the largest civil aviation pilot training school in China. With the rapid development of civil aviation in China, the number of admitted pilot students has multiplied recently. After classroom training, students are sent to the airport training bases to fly on several types of coaching aircrafts. The types of aircrafts and their spare parts are not identical and don't have commonality. For example, base X is the only one accounting for the university's helicopter training, so this base holds all the helicopters the university purchased. So bases make their plan on spare parts individually.

In order to quick repair, upon failure of a particular spare part, maintenance staff will remove the defective one, isolate the failure and replace with another functioning part. These parts are referred as Line Replaceable Units (LRU). The immediate replacement can prevent aircrafts from disruptions caused by part failure to a certain degree. But it's not the case if the demanding LRUs are out of stock. Under such circumstance, the defective LRUs are sent to the central repair shop in the university until they are recovered and back. In very rare conditions, the central repair shop is not able to recover the 
failure and the LRUs whether need to be repaired in other qualified maintenance departments or directly scrapped and reordered in upstream suppliers.

Among each aircraft type, some of the spare parts are attached great importance because its stock-out will deeply impact the flight dispatch decisions. These parts are called critical parts, having great stock-out cost (because downtimes of aircrafts render students from training) on the one hand, usually of high value and with long lead time on the other hand. We can't stock parts as many as possible because the total investment capital in LRUs is limited. The problem of allocating base stock for every item is traditionally treated individually by single item. It turned out to be inefficient because every item tends to have high stock levels.

\section{Modeling}

Considering all the factors aforementioned, a single location multi-item repairable parts inventory model can be applied to tackle the problem of allocating stock in each single base, regardless of the aircraft types. We choose a certain type of aircrafts, and there are many different kinds of spare parts are kept in stock. Only the critical spare parts, the failure of which could result in downtime of aircraft, are taken into consideration in this model.

Notations. Denote set $I$ as the item sequence number of LRU types, and $\mathrm{I}=\{1,2, \ldots\}$. For example, $\mathrm{LRU}_{i}$ stands for LRU of item number $i$. Other notations are defined as follows:

$\mu_{i}=$ the constant failure rate (or demand rate) for $\operatorname{LRU}_{i}, \mu_{i}>0$

$t_{i}=$ the average repair and transport time (or lead time) for $\operatorname{LRU}_{i}, t_{i}>0$

$S_{i}=$ the base stock level for $\mathrm{LRU}_{i}$, waiting to be allocated in the model. $S_{i} \geq 0$

$c_{i}=$ the purchase price of $\operatorname{LRU}_{i}$, also viewed as investment cost, $c_{i}>0$

$C=$ the total cost used to invest in LRUs inventory.

Assumptions. For convenience, the model is established on some fundamental assumptions:

1. The failure process (or demand process) of each single item at the training base are Poisson processes with a deterministic rate and are mutually independent. That is to say, the number of defective spare parts that occurs in a certain period of time follows the identical and independent distribution (i.i.d.) of Poisson.

2. The repair and transport processes (or lead time process) of each single item at the repair shop are i.i.d, too.

3. The repair capacity is always sufficient to meet the demand of repairing. In classical models, researchers often assume the repair capacity is infinite for it's convenient to estimate the number of jobs in system. Because the average queuing length is zero and number of jobs in system equals those in service. However, according to queuing theory, it proved that whenever the average repair rate is much faster than average demand rate, it is the case for the number of jobs in system.

4. The base stock for LRUs is checked by a continuously-reviewed policy and replenished by a one-for-one policy. The replenishment policy is known as the $(S-1, S)$ strategy.

5. Demand for a functional LRU from base stock is fulfilled immediately if available, otherwise it's backordered. The time for removal and installation is ignored in this model.

6. When defective parts are sent to repair, the service follows a first-come and first-service (FCFS) rule. After service, the spare parts are recovered as new.

Objectives and Constraints. System Availability, statistically denoting the percentage of functioning equipments in given period of time, is often used to evaluate the reliability of systems. According to Sherbrooke's research [2,3], average system availability is closely related to the expected backorders. By reliability theory, assuming failures of each LRU on different aircrafts occur independently, an approximated equation of system availability $A$ is obtained as:

$$
A \approx \prod_{i=1}^{I}\left(1-\frac{E B O\left(S_{i}\right)}{N Z_{i}}\right)^{Z_{i}}
$$


In the equation, $N$ denotes the number of aircrafts, and $Z_{i}$ denotes the number of $\mathrm{LRU}_{i}$ installed on each aircraft. The equation can be simplified by taking logarithm on both sides:

$$
A \approx 1-\sum_{i=1}^{I} Z_{i} \frac{E B O\left(S_{i}\right)}{N Z_{i}}=1-\frac{1}{N} \sum_{i=1}^{I} E B O\left(S_{i}\right)
$$

According to the approximation, problems of maximizing the system availability are equivalent to minimizing the aggregate expected backorders. Considering the investment constraints described before, we put forward the objective function and constraints as:

$$
\begin{aligned}
& \min \sum_{i=1}^{I} \operatorname{EBO}\left(S_{i}\right) \\
& \text { s.t. } \sum_{i=1}^{I} c_{i} S_{i} \leq C \\
& S_{i} \in\{0,1, \ldots, n\}
\end{aligned}
$$

It is a non-linear objective function subjected to a linear constraint. Because the decision variables are integral numbers, it is a nonlinear integral programming problem.

Stock Balance Equation. In order to compute EBO, a fundamental stock balance equation is introduced by Sherbrooke.[2,3] It serves as the building blocks of the incoming analysis:

$\mathrm{S}=\mathrm{OH}+\mathrm{DI}-\mathrm{BO}$

For notational convenience, Eq.4 omits index number $i$ in every variable. Specifically, $S$ denotes the base stock; $\mathrm{OH}$ denotes the number of LRUs on hand; $D I$ denotes the number of LRUs due in for repair or transport; $B O$ denotes the number of backorders. The stock balance equation reflects the dynamic balance of these importance variables. All variables are non-negative integrals. Under any circumstance, at least one of variables in $O H$ and $B O$ must be 0 . Table 1 demonstrates some of the possible integers these variables can take, as well as the corresponding probability concerning each incidence.

Table 1 Possible integeral value and corresponding possibilities of $O H, D I$ and $B O$

\begin{tabular}{llll}
\hline Proabability & $\boldsymbol{O H}$ & $\boldsymbol{D I}$ & $\boldsymbol{B O}$ \\
\hline $\boldsymbol{P}\{\boldsymbol{D I}=\boldsymbol{0}\}$ & $S$ & 0 & 0 \\
$\boldsymbol{P}\{\boldsymbol{D I}=\boldsymbol{1}\}$ & $S-1$ & 1 & 0 \\
$\boldsymbol{P}\{\boldsymbol{D I}=2\}$ & $S-2$ & 2 & 0 \\
$\boldsymbol{P}\{\boldsymbol{D I}=\boldsymbol{S}-\boldsymbol{1}\}$ & $S-(S-1)$ & $S-1$ & 0 \\
$\boldsymbol{P}\{\boldsymbol{D I}=S\}$ & 0 & $S$ & 0 \\
$\boldsymbol{P}\{\boldsymbol{D I}=\boldsymbol{S}+\boldsymbol{1}\}$ & 0 & $S+1$ & 1 \\
$\boldsymbol{P}\{\boldsymbol{D I}=\boldsymbol{S}+2\}$ & 0 & $S+2$ & 2 \\
\hline
\end{tabular}

Table 1 only shows part of the possible values of the variables. The table can go on by setting BO any positive integral number. According to the table, we can derive the expected backorders:

$$
\begin{aligned}
& E B O=P\{D I=S+1\}+P\{D I=S+2\} \times 2+\ldots+P\{D I=S+x\} \times x+\ldots, x \in N \\
& \Rightarrow E B O=\sum_{x=0}^{\infty} x P\{D I=S+x\}=(S-x) \sum_{x=0}^{S-1} P\{D I=x\}+E(D I)-S
\end{aligned}
$$

Expected BackOrders. The function of $E B O$ is denoted by the variables of $D I$ and $S$. Because the failed parts arrive the repair shop by a Poisson process of intensity $\mu_{i}$ and stay in the repair system for an average time of $t_{i}$, the steady-state distribution of of number of parts in this queing system is Poisson with mean $\mu_{i} t_{i}$ by Palm's Theorem[6]. That is to say, the pipeline of $D I$ for each $\operatorname{LRU}_{i}$ is a Poisson distribution with mean $\mu_{i} t_{i}$, then:

$$
P\{D I=x\}=\frac{\left(\mu_{i} t_{i}\right)^{x}}{x !} e^{-\mu_{i} t_{i}}, x \in\{0,1,2, \ldots\}
$$


and $E(D I)=\mu_{i} t_{i}$

The Eq.4 can be further simplified as:

$$
E B O=(S-x) \sum_{x=0}^{S-1} P\{D I=x\}+\mu t-S
$$

The equation has only one decision variable $\mathrm{S}$.

The optimazation of $E B O$ under investment constrain can be generated by marginal analysis in the coming sections. The basis for the use of marginal analysis is the deceasing feature and the convexity of $E B O$.

It can be proved that the function of EBO is decreasing and convex for all $\mathrm{S}$ in its domain because:

$$
\begin{aligned}
& \triangle E B O(S)=E B O(S+1)-E B O(S)=-\sum_{x=S+1}^{\infty} P\{D I=x\} \leq 0, S \in\{0,1,2, \ldots\} \\
& \Delta^{2} E B O(S)=\triangle E B O(S+1)-\triangle E B O(S)=P\{D I=S+1\} \geq 0, S \in\{0,1,2, \ldots\}
\end{aligned}
$$

Eq. 9 indicates the function of $E B O$ is decresing for S. A single unit of increase in $\mathrm{S}$ leads to a decrease in $E B O$. While Eq.10 indicates the degree of every single decrease in $E B O$ by increasing $\mathrm{S}$ is gradually reduced.

Marginal Analysis. When it comes back to the objective function and constraints of our problem, a usual and classic solution is marginal analysis. Alternative optimazation techniques include so-called greedy algorithm (actually a systematic way of marginal analysis), Lagrangian Relaxation, and Dantzig-Wolfe Decomposition. However, marginal analysis is preferred in this model for it's concise and convenient to use in small system. The procedures of marginal analysis are:

Step 1: Set every $S_{i}=0$ as a feasible solution for the problem. At this time, compute the original $E B O\left(S_{i}=0\right)=\mu_{i} t_{i}$ and $\sum_{i=0}^{I} E B O\left(S_{i}=0\right)=\sum_{i=0}^{I} \mu_{i} t_{i}, \sum_{i=0}^{I} c_{i} S_{i}=0$.

Step 2: Choose the item of LRUs best worth being increased by one unit, which will lead to the greatest decrease in its EBO. Specifically,

$$
\text { set } \tau_{i}=\left|\frac{\Delta E B O\left(S_{i}\right)}{c_{i}}\right| \text {, and choose the item } k \text { for } k=\arg \max \left\{\tau_{i}\right\} \text {. }
$$

Increase the amount of $S_{k}$ by 1 unit.

Step 3: Compute the objective and check constraint for new feasible solutions. If the investment of total captial constraint is met, stop computation and we will get the lowest possible expected backorder under certain investment constraint; Otherwise go back to step 2.

\section{Application to the case of training base's aircrafts}

Now a single training base of the flight university, have 3 critical items of LRUs in a particular type of aircraft in stock. Planning departments try to establish the base stock for the LRUs under a total capitol investment constraint of 330,000 yuan. In order to both ensure safety and make best use of aircraft, we apply the upper model.

First we gathered historical data of parts failure and its repair service to get parameters of average arriving rate $\mu \mathrm{i}$ and average repair time ti for each item of LRU. These data are demonstrated in Table 2 , together with purchasing prices ${ }^{c_{i}}$ for new LRUs.

Table 2 Pararameters of failure parts arrival/service/purchasing price for LRUi

\begin{tabular}{llll}
\hline Parameter & LRU $_{\boldsymbol{1}}$ & LRU $_{2}$ & LRU $_{3}$ \\
\hline $\boldsymbol{\mu}_{\boldsymbol{i}}$ (unit/year) & 5 & 0.5 & 6 \\
$\boldsymbol{t}_{\boldsymbol{i}}$ (year) & 0.4 & 1 & 0.5 \\
$\boldsymbol{c}_{\boldsymbol{i}}(\mathbf{1 0 , 0 0 0}$ yuan) & 4 & 2 & 6 \\
\hline
\end{tabular}


Then, we compute the expected backorders $\operatorname{EBO}(\mathrm{Si}=0)$ for each LRUi as the first set of results under feasible solutions of $\mathrm{Si}=0$, awaiting further improvement. Following the steps of marginal analysis, we continnue to compute the related parameters such as $\triangle E B O\left(S_{i}\right)$ and $\tau_{i}$ to decide the next item of LRU to be increased in quantity. Repeat the steps and stop the algolorithm until constraint is met, finally we can get data as showed in Table 3.

Table 3 Results of marginal analysis in each step

\begin{tabular}{lccccllll}
\hline Iteration & $\boldsymbol{\tau}_{\boldsymbol{1}}$ & $\boldsymbol{\tau}_{\boldsymbol{2}}$ & $\boldsymbol{\tau}_{\mathbf{3}}$ & $\boldsymbol{S}_{\boldsymbol{1}}$ & $\boldsymbol{S}_{\boldsymbol{2}}$ & $\boldsymbol{S}_{\mathbf{3}}$ & $\sum \boldsymbol{E B O}\left(\boldsymbol{S}_{\boldsymbol{i}}\right)$ & $\sum \boldsymbol{c}_{\boldsymbol{i}} \boldsymbol{S}_{\boldsymbol{i}}$ \\
\hline $\mathbf{0}$ & - & - & - & 0 & 0 & 0 & 5.5 & 0 \\
$\mathbf{1}$ & 0.216 & 0.196750 & 0.158367 & 1 & 0 & 0 & 4.6353 & 4 \\
& 175 & & & & & & & \\
$\mathbf{2}$ & 0.148525 & 0.045100 & 0.133467 & 2 & 0 & 0 & 4.0412 & 8 \\
$\mathbf{3}$ & 0.080875 & 0.007200 & 0.096117 & 2 & 0 & 1 & 3.091 & 14 \\
$\mathbf{4}$ & 0.035775 & 0.000900 & 0.058767 & 2 & 0 & 2 & 2.2902 & 20 \\
$\mathbf{5}$ & 0.013225 & 0.0001105 & 0.03075 & 2 & 0 & 3 & 1.7135 & 26 \\
$\mathbf{6}$ & 0.010218 & $0.0000 \ldots$ & 0.00553 & 2 & 0 & 4 & 1.3609 & 32 \\
\hline
\end{tabular}

At Step 6, we have to stop the analysis since the total capital investment in LRUs has risen up to 320,000 yuan. The left 10,000 is not enough to buy any single unit among the 3 items. The set of optimal solutions in the question is obtained as $\{\mathrm{S} 1=2, \mathrm{~S} 2=0, \mathrm{~S} 3=4\}$. The agregate expected number of backorders EBO has been improved by 6 times in investing 2 units on LRU1 and 4 units on LRU2 from the orginal feasible solutions.

\section{Conclusions}

In this paper, we give a thorough introduction of classic single location, multi-item model frequently applied in spare parts inventory control. The model then is employed to deal with the problem from a training base in a flight university. Obviously it is superior to conventional methods, most of which view the inventory optimization in an isolated single-item way. In future research, there are several directions to follow to deeply study the case. Firstly, for convenience, we only take 3 critical LRUs in the research to compute. However, there are actually hundreds of thousands items of LRUs to be considered on their base stock levels in forecasting and decision-making scenarios. Optimization techniques in large-system problem can be introduced. Secondly, the problem can be extended and studied in a multi-echelon or multi-indenture inventory system in the future.

\section{References}

[1] Feeney.G.J. and Sherbrooke. C.C, The (S-1, S) inventory policy under compound Poission demand. Management Science. 27 (1966) 391-411.

[2] Sherbrooke. C.C, METRIC: a multi-echelon technique for recoverable item control. Operations Research. 16 (1968) 122-141.

[3] Sherbrooke. C.C, Optimal inventory modeling of systems: multi-echelon technique, 2nd ed., New York, 2004 .

[4] Muckstadt, J.A., A model for a multi-item, multi-echelon, multi-indenture inventory system. Management Science 20, (1973)472 - 481.

[5] Slay, F.M., Vari-metric: an approach to modeling multi-echelon resupply when the demand process is Poisson with gamma prior. Report AF301-3, Logistics Management Institue, Washington D.C., 1984.

[6] Palm, C., Analysis of the Erlang traffic formula for busy-signal arrangements, Ericsson Technics, 4, (1938) 204-212 